\title{
TopEVM: Using Co-occurrence and Topology Patterns of Enzymes in Metabolic Networks to Construct Phylogenetic Trees
}

\author{
Tingting Zhou ${ }^{1,2}$, Keith C.C. Chan ${ }^{2}$, and Zhenghua Wang ${ }^{1}$ \\ ${ }^{1}$ National Laboratory for Paralleling and Distributed Processing, \\ National University of Defense Technology, Changsha, Hunan, 410073, P.R. China \\ Tingting. Zhoulive.com, zhhwang188@sina.com \\ ${ }^{2}$ Department of computing, The Hong Kong Polytechnic University, Hong Kong, China \\ cskcchan@inet.polyu.edu.hk
}

\begin{abstract}
Network-based phylogenetic analysis typically involves representing metabolic networks as graphs and analyzing the characteristics of vertex sets using set theoretic measures. Such approaches, however, fail to take into account the structural characteristics of graphs. In this paper we propose a new pattern recognition technique, TopEVM, to help representing metabolic networks as weighted vectors. We assign weights according to co-occurrence patterns and topology patterns of enzymes, where the former are determined in a manner similar to the $T f$-Idf approach used in document clustering, and the latter are determined using the degree centrality of enzymes. By comparing the weighted vectors of organisms, we determine the evolutionary distances and construct the phylogenetic trees. The resulting TopEVM trees are compared to the previous $N C E$ trees with the NCBI Taxonomy trees as reference. It shows that TopEVM can construct trees much closer to the NCBI Taxonomy trees than the previous NCE methods.
\end{abstract}

Keywords: TopEVM, phylogenetic analysis, metabolic network, co-occurrence pattern, document clustering, topology pattern, degree centrality, evolutionary distance.

\section{Introduction}

The objective of phylogenetic analysis is to reconstruct the evolutionary relationship among different species and to display them in a tree-structured model called a phylogenetic tree [1]. Applications include the design of new drugs and the reconstruction of the history of infectious diseases [2]. Most previous research [3] in this area has been based on sequence alignment but these sequence-based approaches are easily influenced by horizontal gene transfer (HGT) [4,5]. An alternative to this is network-based phylogenetics analysis, which compares the homogeneous biological networks of organisms. They often make use of metabolic networks and take the quantified difference between these networks as the evolutionary distance. 
A metabolic network is a hierarchical, graph-represented abstract of an actual metabolism. Composed of thousands of metabolites, enzymes, reactions and the relationships among them, global metabolic networks are too large and complicated to be compared element by element. So, for comparison purposes, the vertex sets of graphs, rather than the entire graph, is common to use. In such cases, the evolutionary distances are determined by applying set theoretic measures [6-10]. For example, Aguilar et al [11] treat the organisms as enzyme sets from the view of metabolism, building a binary vector for each organism according to the presence or absence of the enzymatic functions. Using the NCE (Number of Common Enzymes) method and a normalized Hamming distance, they construct phylogenetic trees by creating a distance matrix for each metabolic class. Forst et al [8] construct 'clean' metabolite-reaction bipartite graphs to represent metabolic networks. Using the Jaccard distance as the evolutionary distance measure, they construct the distance matrix by taking organisms as reaction sets. Tohsato [7] consider metabolic networks as enzymatic reaction sets. Also using the Jaccard distance, she determines the evolutionary distance matrix and constructed phylogenetic trees. One drawback of such set-theoretic methods is that they do not usually take into account the edge information, and therefore they do not have enough topological characteristics for the network comparison, especially the topological importance of vertices $[9,12,13]$.

In this paper we propose a new pattern recognition technique, TopEVM, for use in phylogenetic analysis. TopEVM avoids a common drawback of set-theoretic methods in that it takes account of the structural characteristics of graphs by representing metabolic networks as weighted vectors. We assign the weights based on the co-occurrence and topology patterns of enzymes in organisms, where the co-occurrence patterns are determined using a method similar to the $T f$-Idf approach in the document clustering and the enzyme topology patterns are determined according to the degree of centrality of enzymes. By comparing the weighted vectors of organisms, we determine the evolutionary distance matrices for the construction of phylogenetic trees. Comparing to the previous set-theoretic methods, TopEVM can produce phylogenetic trees closer to the taxonomy trees of NCBI.

The remainder of this paper is organized as follows. Section 2 elaborates the TopEVM approach. Section 3 describes our experiments and results. Section 4 provides conclusion and outlines directions for the future work.

\section{TopEVM: Constructing Phylogenetic Trees by Using Enzyme Co-occurrence and Topology Patterns}

In this section we describe the operation of the TopEVM approach, which proposes the use of a frequency weighting scheme and a topological vector. This approach proceeds from the observation that it is possible to regard the construction of species trees in phylogeny as similar to the process of distance-based clustering of organisms which may in turn be seen as analogous to document clustering, with an organism as a document and an enzyme as a term. This allows us to apply feature extraction approaches and the hierarchical clustering methods to the construction of phylogenetic trees. 


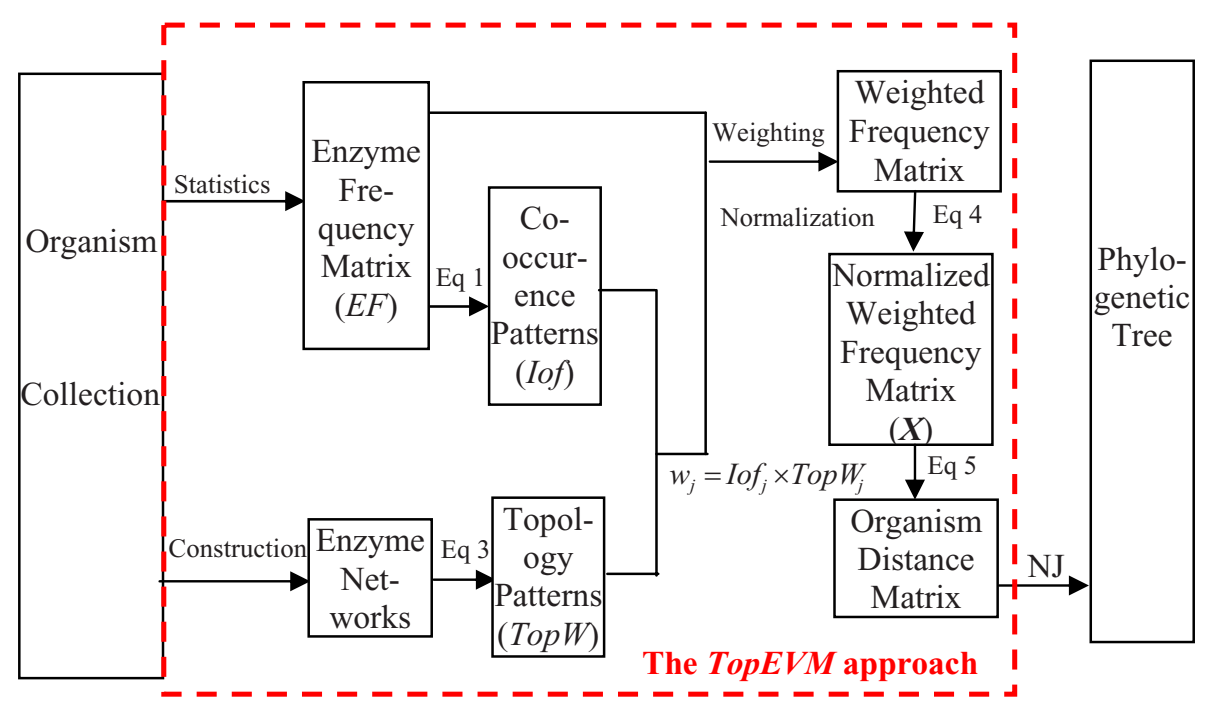

Fig. 1. The TopEVM approach

The first step in the TopEVM approach is to set up a matrix to record how frequently an enzyme occurs in a given collection of organisms, and extract enzyme co-occurrence patterns according to this matrix. By analogy with the $T f$-Idf weighting scheme used in document clustering, we define Inverse organism frequency (Iof), a weight vector, to extract enzyme co-occurrence patterns. In the second step TopEVM uses a topological weight vector, Top $W$, to extract topologically important enzymes, the enzyme topology patterns, for use as features. This is done by representing metabolic networks as enzyme graphs and then counting the degree centrality, one measure of topological importance, of enzymes. The next step is to normalize the enzyme co-occurrence and topology weighting schemes. These are then used to convert the original frequency matrix into a new matrix in which rows denote the final Topology-weighted Enzyme Vector Model (TopEVM) of organisms. Finally a distance matrix is established by comparing the TopEVM of organisms with Soergel Distance as the distance measure. The distance matrix is used to construct the phylogenetic trees by use of some distance-based clustering approach, e.g., Neighbor Joining (NJ) method. Figure 1 shows the flow of the entire procedure.

\subsection{Inverse Organism Frequency: Extracting Enzyme Co-occurrence Patterns}

The first step to extract the co-occurrence patterns of enzymes is to set up a matrix to record how frequently an enzyme occurs in a given collection of organisms. For this purpose, we define Enzyme Vector Space to denote the organism-enzyme frequency matrix and Enzyme Vector Model to denote the organisms as enzyme frequency vectors. It should be noted that we make two assumptions in the definitions. First, we assume that 
enzymes are arranged in the ascending order of their EC numbers ${ }^{1}$ and the order is kept constant. Second, we assume that organisms in the collection are arranged in an arbitrary but constant order.

Enzyme Vector Space. Let $O$ be a collection of $m$ organisms $o_{i}, i=1 . . m$. Let $E$ be all the $n$ enzymes $e_{j}, j=1 . . n$, of at least one organism in $O$. The Enzyme Vector Space $O^{m n}$ is defined through the organism-enzyme frequency matrix, $E F$, where $e f_{i j}$ is the frequency of the $j^{\text {th }}$ enzyme, $j=1 . . n$, in the $i^{\text {th }}$ organism.

Enzyme Vector Model. Given an enzyme vector space $O^{m n}$ and its organism-enzyme frequency matrix, $E F$, the enzyme frequency vector for the $i^{t h}$ organism $o_{i}$ is defined as the $i^{\text {th }}$ row of $E F$. We call this representation of organisms as Enzyme Vector Model.

Document clustering generally assumes that the total term frequency is not always indicative of a term's information content. To account for this disparity, the Inverse document frequency (Idf) weighting scheme is often applied [14]. We find a similar situation when we compare the enzyme frequency vectors of organisms. That is to say, the frequency of an enzyme appeared in all the organisms cannot be assumed to indicate its information content. To deal with this, we apply a weighting scheme in this study, which is similar to the $I d f$ weighting scheme. We call this the Inverse organism frequency (Iof) weighting scheme and define it as follows.

Organism Frequency. Given an enzyme vector space $O^{m n}$ and its organism-enzyme frequency matrix $E F$, the Organism Frequency $\left(o f_{j}\right.$ ) of a given enzyme $e_{j}, j=1 . . n$, is defined as the number of organisms that contain the enzyme $e_{j}$.

Inverse Organism Frequency. Given a enzyme vector space $O^{m n}$ and its organism-enzyme frequency matrix $E F$, the Inverse organism frequency $\left(I o f_{j}\right)$ of a given enzyme $e_{j}, j=1 . . n$, is defined as the logarithm of the quotient of dividing the total organism number by its organism frequency $\left(o f_{j}\right)$. That is,

$$
I o f_{j}=\log \left(m / \sum_{i} I_{\left(e f_{i j}>0\right)}\right)
$$

where $I_{(\cdot)}$ denotes the indicator function, $I_{(\text {cond })}=\left\{\begin{array}{ll}1 & \text { if.cond.is. fulfilled } \\ 0 & \text { otherwise }\end{array}\right.$, and $\sum_{i} I_{\left(e_{i j}>0\right)}$ is the organism frequency of $e_{j}$, namely $o f_{j}$.

Once $I o f_{j}$ has been assigned to enzyme $e_{j}$, the original frequency of enzyme $e_{j}$ in $\operatorname{organism} o_{i}$, namely $e f_{i j}$, can be transformed into a new weighted frequency $e f_{i j}$,

$$
e f_{i j}{ }^{\prime}=I o f_{j} \cdot e f_{i j}
$$

Since the Iof weighting scheme gives lower weights to the enzymes found in a large number of organisms and higher weights to those found in fewer organisms, the Iof weights emphasize organism-specific enzymes.

\footnotetext{
${ }^{1}$ The EC (Enzyme Commission) number is a numerical classification scheme for enzymes.
} 


\subsection{Topology Weight: Extracting Enzyme Topology Patterns}

Some enzymes in a metabolic network will have a higher average connectivity than others [15]. On the assumption that this higher average connectivity represents topological important information, we define Topology Vector Space to denote the organism-enzyme topology matrix and Topology weight (TopW) weighting scheme to select more strongly connected enzymes.

Topology Vector Space. Let $\boldsymbol{R}$ be a collection of $m$ metabolic networks. $r_{i}$ is constructed for the $i^{\text {th }}$ organism $o_{i}, i=1 . . m$. Let $\boldsymbol{E}$ be the collection of all the $n$ enzymes $e_{j}$, $j=1 . . n$, which are contained by at least one metabolic network in $\boldsymbol{R}$. The Topology Vector Space $\boldsymbol{R}^{\boldsymbol{m} \boldsymbol{n}}$ is defined through the organism-enzyme topology matrix, $\boldsymbol{T}$, where $t_{i j}$ is the topological importance of the $j^{\text {th }}$ enzyme in the metabolic network of the $i^{\text {th }}$ organism, $j=1 . . n$.

In this study, the degree centrality, the number of direct neighbors of a node [16], is regarded as the measure of the node's topological importance. In order to distinguish the absent enzymes from the present enzymes with degree as ' 0 ', we assign the degree centrality of the absent enzymes as ' -1 ' in the topology matrix $\boldsymbol{T}$.

Topology Weight. Given a topology vector space $R^{m n}$ and its organism-enzyme topology matrix $T$, the Topology weight $\left(\right.$ Top $\left.W_{j}\right)$ of the given enzyme $e_{j}, j=1 . . n$, is defined as:

$$
T o p W_{j}=\sum_{i}\left(t_{i j} \cdot I_{\left(t_{i j} \geq 0\right)}\right) / \sum_{i} I_{\left(t_{i j} \geq 0\right)}
$$

where $I_{(\cdot)}$ is an indicator function defined as in Eq 1, and $\sum_{i} I_{\left(t_{i j}>0\right)}$ is the total number of metabolic networks in $\boldsymbol{R}$ containing enzyme $e_{j}$.

The Top $W$ weighting scheme gives higher weights to the enzymes with higher average degree, which strengthens the importance of more highly-connected enzymes.

\subsection{Normalization: Eliminating the Influence of Vector Length on Distance}

The difference of the vector length can influence the calculation of the distance between vectors. Iof and Top $W$ weighting schemes help select the 'important' enzymes as the features of organisms, but result in the organism vectors with different lengths. Therefore, it is necessary to normalize the weighted organism vectors before calculating the distance between them.

Let $\boldsymbol{X}$ denotes the weighted and normalized organism-enzyme frequency matrix, where the element $x_{i j}, i=1 . . m, j=1 . . n$, is given by

$$
x_{i j}=\frac{w_{j} \times f_{i j}}{\sqrt{\sum_{k}\left(w_{k} \times f_{i k}\right)^{2}}}, \quad w_{j}=I o f_{j} \times \operatorname{Top}_{j}
$$

The rows in $\boldsymbol{X}$ are the final representative of organism vectors. 


\subsection{Soergel Distance: Calculating the Evolutionary Distance}

Soergel distance is one of the distance measures which are commonly used to calculate the evolutionary distance, a crucial measure of the similarity of organism vectors. It has the advantages that its range is limited to $0 \sim 1$ and it obeys the triangular inequality [17].

Suppose $\boldsymbol{X}_{\boldsymbol{A}}$ and $\boldsymbol{X}_{\boldsymbol{B}}$ are two vectors of equal length $n$, the Soergel Distance between them is defined as:

$$
D_{A, B}=\frac{\sum_{j=1}^{n}\left|x_{j A}-x_{j B}\right|}{\sum_{j=1}^{n} \max \left(x_{j A}, x_{j B}\right)}
$$

The distance matrix can be established by calculating the Soergel distance between organism vectors pair-wisely. It can be used to construct the phylogenetic trees using a suitable distance-based clustering algorithm, e.g. Neighbor-joining $(N J)$ method.

\section{Experiments and Results}

In this study, enzyme and reaction data are obtained from the database created by $\mathrm{Ma}$ and Zeng [18]. The Ma and Zeng database consists of five tables: reaction, enzyme, react, connect and organism, and contains 3663 enzymes and 107 organisms (8 Eukaryotes, 83 Bacteria and 16 Archaea) in total. We acquire enzyme frequency information from enzyme, and construct the enzyme graphs for each organism from enzyme and reaction.

Although the TopEVM approach is capable of dealing with large collections of organisms, for the sake of concision, in this explanation we select only eight organisms: rno, mmu, afu, mja, nme, hin, lin and bsu.

Table 1 lists the details of these 8 organisms: their ID in KEGG database (KEGG ID), their full name(Organism), the Kingdom they belong to (Kingdom) their ID in NCBI Taxonomy [19] (NCBI Tax Id), and the number of enzymes they contain $\left(N_{E}\right)$.

Table 1. The details of the 8 organisms

\begin{tabular}{ccccc}
\hline KEGG & Organism & Kingdom & NCBI & \multirow{2}{*}{ Tax ID } \\
ID & Rattus norvegicus & Eukaryota & 487 & 416 \\
\hline rno & Mus musculus & Eukaryota & 727 & 470 \\
mmu & Archaeoglobus fulgidus & Archaea & 1423 & 277 \\
afu & Aethanococcus jannaschii & Archaea & 1642 & 244 \\
mja & Neisseria meningitides & ProteoBacteria & 2190 & 369 \\
nme & Haemophilus influenzae & ProteoBacteria & 2234 & 386 \\
hin & Listeria innocua & Bacteria Firmicute & 10090 & 388 \\
lin & Bacillus subtilis & Bacteria Firmicute & 10116 & 504 \\
bsu & \multicolumn{5}{c}{}
\end{tabular}




\subsection{Distribution of the Iof Weights}

According to the definitions in Section 2.1, omitting the absent enzymes in all of the 8 organisms, we calculate the Iof weight vector of length 1063. The Iof weights values distribute over 8 points. Table 2 displays the value of $N_{E}$ along the Iof Weights. Nearly $30 \%$ enzymes have the highest Iof weights. $2.1 \%$ enzymes will be neglected for their Iof importance are 0. Moreover, there are around $60 \%$ enzymes with the Iof value over 1 , and $40 \%$ between 0 and 1 . Since the Iof weighting scheme gives lower weights to the enzymes occurring in a large number of organisms, it comes that the lower the Iof value is, the more organisms the enzyme spreads in. This observation also confirms the conclusion of Liu et al [13]. That is, most of the enzymes occur in several organisms they prefer, while only few enzymes occur in most of the studied organisms.

Table 2. The statistics of the number of enzymes along the Iof weights

\begin{tabular}{ccccccccc}
\hline Iof Weight & $\mathbf{2 . 0 8}$ & $\mathbf{1 . 3 9}$ & $\mathbf{0 . 9 8}$ & $\mathbf{0 . 6 9}$ & $\mathbf{0 . 4 7}$ & $\mathbf{0 . 2 9}$ & $\mathbf{0 . 1 3}$ & $\mathbf{0}$ \\
\hline Num Enzymes & 318 & 310 & 102 & 118 & 71 & 85 & 36 & 23 \\
Percentage $(\%)$ & 30 & 29.1 & 9.6 & 11.1 & 6.7 & 8.0 & 3.4 & 2.1 \\
\hline
\end{tabular}

\subsection{Distribution of the TopW Weights}

In order to calculate the TopW weights, we represent the enzyme networks upon the following principles: vertices denote individual enzymes and arcs denote the relationships between them; if one enzyme's product is the substrate of another enzyme, then there's an arc directed from the former enzyme to the latter. The bidirectional arc is replaced by two individual arcs with opposite direction.

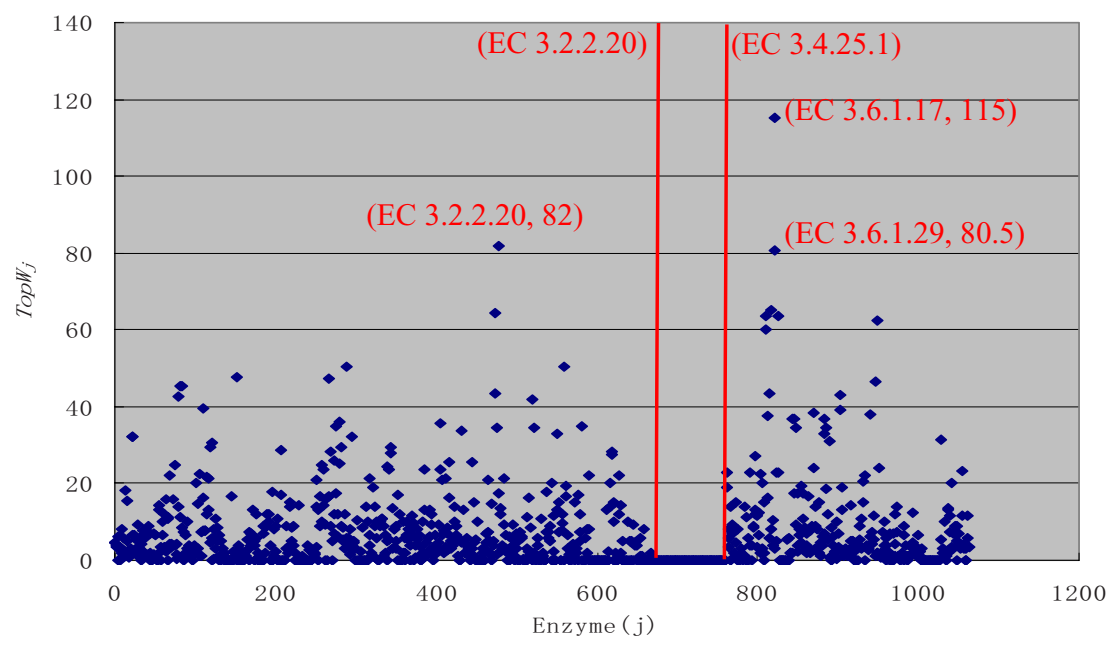

Fig. 2. Distribution of the Top $W$ weights. The $x$-coordinates denote the ordered enzymes. The $y$-coordinates denote the corresponding Top $W$ weight value of the enzyme. The coordinates of the top 3 enzymes are marked on the figure. The continuous range between two vertical dash lines denotes the range of enzymes whose Top $W$ weight is 0 . 
Table 3. The enzymes with the top 8 Top $W$ value

\begin{tabular}{ccccccccc}
\hline Order & $\mathbf{1}$ & $\mathbf{2}$ & $\mathbf{3}$ & $\mathbf{4}$ & $\mathbf{5}$ & $\mathbf{6}$ & $\mathbf{7}$ & \multicolumn{1}{c}{$\mathbf{8}$} \\
\hline Enzyme & 3.6 .1 .17 & 2.7 .4 .10 & 3.6 .1 .29 & 3.6 .1 .15 & 2.7 .4 .6 & 3.6 .1 .3 & 3.6 .3 .1 & 4.6 .1 .1 \\
Top $\boldsymbol{W}$ & 115 & 82 & 80.5 & 65 & 64.25 & 63.5 & 63.5 & 62.3 \\
\hline
\end{tabular}

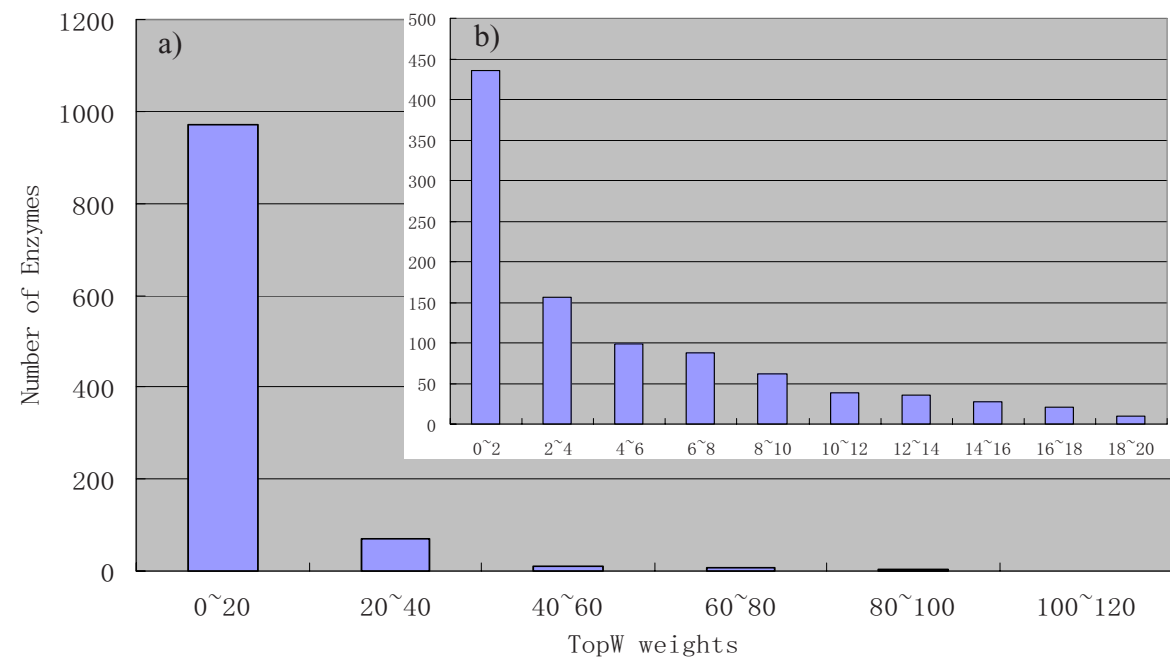

Fig. 3. Distribution of the number of enzymes along the Top $W$ weights It contains two diagrams. Fig 3a) shows the distribution of enzymes all over the range of the TopW weights. Fig 3b) expands the Top $W$ weight range of $0 \sim 20$.

We construct the Top $W$ weight vector for the ordered enzyme array on the basis of the definitions in Section 2.2. Fig. 2 shows the distribution of the TopW weight values. Most of the TopW weights are small but several are very big. For example, dinucleoside tetraphosphatase (EC 3.6.1.17)'s TopW weight is 115, AMP phosphotransferase (EC 2.7.4.10)'s is 82, and bis(5'-adenosyl)-triphosphatase (EC 3.6.1.29)'s is 80.5. This observation indicates that as a topology pattern, the Top $W$ weights of few enzymes are high, while that of most enzymes are low. We also notice that from $E C$ 3.2.2.20 to $E C$ 3.4.25.1, there is a gap in which 110 enzymes have Top $W$ values as 0 . They are part of glycosyl hydrolases (EC 3.2.-.-), and all of the hydrolases acting on ether bonds (EC 3.3.-.-) as well as peptide bonds (EC 3.4.-.-). It is mostly due to either the large absence of the enzymes or their possible isolation.

Table 3 displays the enzymes with the top 8 Top $W$ weights. It shows that the $h y$ drolases acting on acid anhydrides (EC 3.6.-.-) have more connection, which means hydrolases may be more topologically important than the enzymes with other function.

Fig 3 shows the distribution of the number of enzymes along the Top $W$ range. It can be seen in Fig 3a) that more than $90 \%$ of enzymes are found within the Top $W$ range of 
0 20 (the first tallest bar). Fig 3b) expands the first bar of Fig 3a), and displays its details of the distribution. As is shown in Fig 3b), there are 436 enzymes in the TopW range of $0 \sim 2$. The number is nearly $41 \%$ of the total number of the enzymes. It indicates that most of nodes have very low connectivity but a handful of nodes (hubs) have much higher connectivity in the constructed enzyme networks. This result is in accordance with the scale free property of metabolic networks, and shows that enzymes become topologically different during evolution [15].

a)

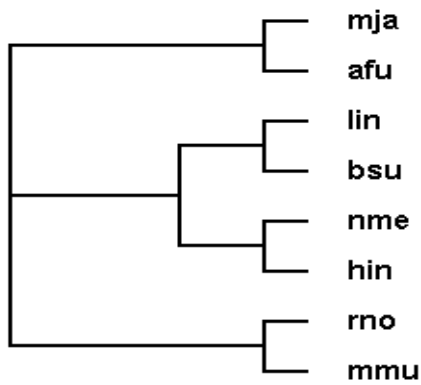

b)

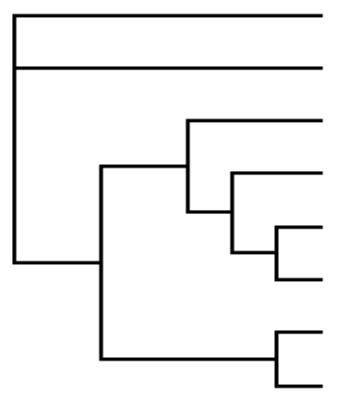

rno

mmu

bsu

hin

lin

nme

afu

mja c)

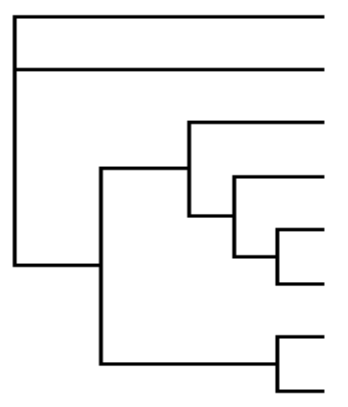

mnu

bsu

hin

lin

mja

afu

nme

Fig. 4. The comparison of a) the NCBI tree, b) the TopEVM tree, and c) the NCE tree

\subsection{Construction and Evaluation of TopEVM Phylogenetic Tree}

We calculate the distance for each organism pair by use of Soergel distance, and obtain the distance matrix (Table 4) for constructing the phylogenetic tree. With the help of the Phylip [20] package, we use NJ (Neighbor Joining) method to do the construction. The resulting tree is rootless, which is displayed as Fig 5b) by use of TreeView [21]. We also obtain the phylogenetic tree from NCBI Taxonomy (Fig 5a) as the reference, and construct trees using the $N C E$ method (Fig 5c) for evaluation.

As is shown in the TopEVM tree, the two Archaea $a f u$ and $m j a$ are grouped together undoubtedly, which is in line with the taxonomy from NCBI, and so do the two Eukaryotes rno and $m m u$. In the NCE tree, although rno and $m m u$ are grouped together, the 4 Bacteria and 2 Archaea are mixed up.

We use TOPD/FMTS [22] to evaluate the similarities of trees. This software is complemented with a randomization analysis to test the null hypothesis that the similarity 
Table 4. The resulting distance matrix of the 8 organisms upon TopEVM

\begin{tabular}{lllllllll}
\hline & тти & rno & afu & nme & mja & hin & lin & bsu \\
\hline mmu & 0.0000 & 0.1079 & 0.7201 & 0.7224 & 0.6754 & 0.6905 & 0.7468 & 0.6790 \\
rno & 0.1709 & 0.0000 & 0.7613 & 0.7793 & 0.7520 & 0.7613 & 0.8020 & 0.7073 \\
afu & 0.7201 & 0.7613 & 0.0000 & 0.2737 & 0.4409 & 0.4911 & 0.6667 & 0.5287 \\
nme & 0.7224 & 0.7793 & 0.2737 & 0.0000 & 0.4471 & 0.3951 & 0.6266 & 0.5617 \\
mja & 0.6754 & 0.7520 & 0.4409 & 0.4471 & 0.0000 & 0.2395 & 0.5382 & 0.3533 \\
hin & 0.6905 & 0.7613 & 0.4911 & 0.3951 & 0.2395 & 0.0000 & 0.4438 & 0.3129 \\
lin & 0.7468 & 0.8020 & 0.6667 & 0.6266 & 0.5382 & 0.4438 & 0.0000 & 0.2486 \\
bsu & 0.6790 & 0.7073 & 0.5287 & 0.5617 & 0.3533 & 0.3129 & 0.2486 & 0.0000 \\
\hline
\end{tabular}

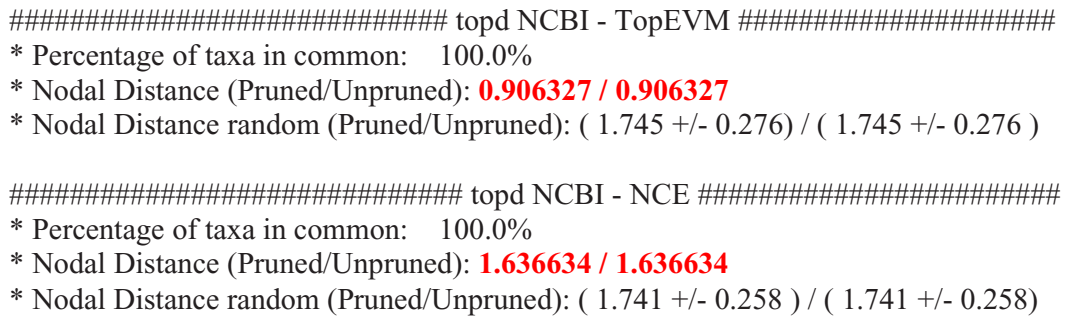

Fig. 5. The comparison result of the TopEVM tree and the NCE tree with the NCBI tree as reference

between two trees is not better than chance. With the NCBI tree as the reference, the comparison result of the TopEVM tree and the NCE tree is showed in Fig 6, which shows the TopEVM tree is closer to the NCBI tree with a less Nodal Distance [23] as 0.9.

\section{Conclusion and Future Work}

This paper proposes a new pattern recognition technique, TopEVM, which represents the metabolic networks as weighted vectors. By calculating the distances among these weighted vectors, evolutionary distance matrices are determined for the construction of phylogenetic trees. Comparing to the previous set-theoretic methods, our TopEVM method results a phylogenetic tree closer to the taxonomy tree of NCBI, which shows TopEVM can be a very useful approach for the network-based phylogenetic analysis.

Nevertheless, our experiments so far have considered only the $T f$-Idf weighting scheme to integrate enzyme's frequency content. It is hard to say that there is no other weighting scheme which is more suitable. Besides, among the extensive topological indices, we only considered the degree centrality; we would also like to consider more topological information for improving our model further. 
Acknowledgments. We thank Dr. Hong-Wu Ma and Prof. Dr. An-Ping Zeng for sharing their revised metabolic database. We also thank Dr. Puigbò for the direction on the use of TOPD/FMTS. This work is supported by the National Natural Science Foundation of China (6077 3021).

\section{References}

1. Husmeier, D.: Introduction to Statistical Phylogenetics. In: Husmeier, D., Richard Dybowski, R., Roberts, S. (eds.) Probabilistic Modeling in Bioinformatics and Medical Informatics. Springer, Heidelberg (2006)

2. Holmes, E.C., et al.: Using Phylogenetic Trees to Reconstruct the History of Infectious Disease Epidemics. In: Harvey, P. (ed.) New Uses for New Phylogenies. Oxford University Press, Oxford (1996)

3. Ludwig, W., Schleifer, K.: Bacterial Phylogeny Based on 16S and 23S rRNA Sequence Analysis. FEMS Microbiol Rev. 15(2-3), 155-173 (1994)

4. Wolf, Y.I., et al.: Genome Trees and the Tree of Life. Trends in Genetics 18(9), 472-479 (2002)

5. Pal, C., Papp, B., Lercher, M.J.: Adaptive Evolution of Bacterial Metabolic Networks by Horizontal Gene Transfer. Nature Genetics 37(12), 1372-1375 (2005)

6. Ebenhöh, O., Handorf, T., Heinrich, R.: A Cross Species Comparison of Metabolic Network Functions. Genome Informatics 16(1), 203-213 (2005)

7. Tohsato, Y.: A Method for Species Comparison of Metabolic Networks Using Reaction Profile. IPSJ Digital Courier 2(0), 685-690 (2006)

8. Forst, C.V., Flamm, C., Hofacker, I.L., Stadler, P.F.: Algebraic Comparison of Metabolic Networks, Phylogenetic Inference, and Metabolic Innovation. BMC Bioinformatics 7(1), 67-78 (2006)

9. Zhou, T., Chan, C., Pan, Y., Wang, Z.: An Approach for Determining Evolutionary Distance in Network-Based Phylogenetic Analysis. In: Măndoiu, I., Sunderraman, R., Zelikovsky, A. (eds.) ISBRA 2008. LNCS (LNBI), vol. 4983. Springer, Heidelberg (2008)

10. Ma, H.W., Zeng, A.P.: Phylogenetic Comparison of Metabolic Capacities of Organisms at Genome Level. Molecular Phylogenetics and Evolution 31(1), 204-213 (2004)

11. Aguilar, D., et al.: Analysis of Phenetic Trees Based on Metabolic Capabilities Across the Three Domains of Life. Journal of Molecular Biology 340(3), 491-512 (2004)

12. Zhu, D., Qin, Z.S.: Structural Comparison of Metabolic Networks in Selected Single Cell Organisms. BMC Bioinformatics 6(8) (2005)

13. Liu, W., Lin, W., Davis, A., Jordan, F., Yang, H., Hwang, M.: A Network Perspective on the Topological Importance of Enzymes and Their Phylogenetic Conservation. BMC Bioinformatics 8(121) (2007)

14. Aizawa, A.: An Information-theoretic Perspective of Tf-idf Measures. Information Processing and Management 39(1), 45-65 (2003)

15. Light, S., Kraulis, P., Elofsson, A.: Preferential Attachment in the Evolution of Metabolic networks. BMC Genomics 6(1), 159 (2005)

16. Aittokallio, T., Schwikowski, B.: Graph-based Methods for Analyzing Networks in Cell Biology. Briefings in Bioinformatics 7(3), 243 (2006)

17. Willett, P., Barnard, J.M., Downs, G.M.: Chemical Similarity Searching. J. Chem. Inf. Comput. Sci. 38(6), 983-996 (1998) 
18. Ma, H.W., Zeng, A.P.: Reconstruction of Metabolic Networks from Genome Data and Analysis of Their Global Structure for Various Organisms. Bioinformatics 19(2), 270-277 (2003)

19. NCBI Taxonomy, http://www.ncbi.nlm.nih.gov/Taxonomy/

20. Phylip, http://evolution.genetics.washington.edu/phylip.html

21. TreeView, http://taxonomy.zoology.gla.ac.uk/rod/treeview.html

22. Puigbo, P., Garcia-Vallve, S., McInerney, J.O.: TOPD/FMTS: A New Software to Compare Phylogenetic Trees. Bioinformatics 23(12), 1556 (2007)

23. Bluis, J., Shin, D.G.: Nodal Distance Algorithm: Calculating a Phylogenetic Tree Comparison Metric. In: Proceedings of third IEEE Symposium on Bioinformatics and Bioengineering (2003) 